\title{
Effects of repeated deep-frying on fatty acid profiles of potato fries and frying oils: soybean oil, canola oil and their 1:1 blend
}

\author{
Michelle Ting Yun Yeo ${ }^{1}$, Xinyan $\mathrm{Bi}^{1}$ \& Christiani Jeyakumar Henry ${ }^{1,2^{*}}$ \\ ${ }^{1}$ Clinical Nutrition Research Centre (CNRC), Singapore Institute of Food and \\ Biotechnology Innovation (SIFBI), Agency for Science, Technology and Research \\ (A*STAR), Singapore; ${ }^{2}$ Department of Biochemistry, National University of Singapore, \\ Singapore
}

\begin{abstract}
Introduction: This study looked at the fatty acid composition changes in potato fries fried in three different types of oils, namely soybean oil (SO), canola oil (CO), and a 1:1 blend of soybean oil and canola oil (SCO), throughout an intermittent frying process of 80 batches in five consecutive days. The study also examined the fatty acid composition changes in SO, $\mathrm{CO}$ and SCO during the frying process. Methods: Fat from potato fries (extracted by Soxtec system) and oil samples from the corresponding frying oil were analysed by gas chromatography-mass spectrometer (GC-MS) to examine the fatty acid profile changes during the deep-frying process. Results: Linoleic acid (LA) and $\alpha$-linolenic acid (ALA) in all three oils decreased, while oleic acid (C18:1), stearic acid (C18:0), palmitic acid (C16:0) and octanoic acid (C8:0) increased. Formation of C18:1 trans fatty acid was observed as the frying time increased. The fatty acid composition of the potato fries was consistent with the fatty acid composition of the corresponding frying oils. Conclusion: Our results showed that blending soybean oil and canola oil did not significantly improve the frying stability of the resulting oil in terms of fatty acid profile. Due to the formation of trans fatty acids and the decrease in polyunsaturated fatty acids, our study also recommends not to use the same frying oil repeatedly and not to consume food products cooked in reused oil.
\end{abstract}

Keywords: Deep frying, reused oil, fatty acid, potatoes

\section{INTRODUCTION}

It has been reported that long chain omega-3 polyunsaturated fatty acids (PUFAs), including $\alpha$-linolenic acid (ALA), eicosapentaenoic acid (EPA), and docosahexaenoicacid(DHA)areimportant to help protect against cardiovascular disease (CVD), neurodegeneration, and inflammation (Calder, 2006; Rajaram,
2014). Consumption of dietary omega-3 PUFAs is necessary, as the human body requires them for good health. While EPA and DHA are obtained largely from marine-based food, ALA is found mainly in plant oils such as flaxseed, soybean oil (SO), and canola oil (CO).

Since oil is an excellent medium for heat transfer, food is swiftly cooked

\footnotetext{
*Corresponding author: Professor Christiani Jeyakumar Henry

Clinical Nutrition Research Centre (CNRC), Singapore Institute of Food and Biotechnology Innovation (SIFBI), Agency for Science, Technology and Research (A*STAR), 14 Medical Drive \#07-02,

Singapore 117599, Singapore

Tel: +65 64070793 Email: jeya_henry@sifbi.a-star.edu.sg

doi: https://doi.org/10.31246/mjn-2020-0011
} 
when it is plunged into oil (Alvis et al., 2009). However, during the deep-frying process, multiple complex reactions, including oxidation, polymerisation, and hydrolysis occur in the frying oil and its fried product (Choe \& Min, 2007). As a result, components such as free fatty acids and trans isomers are formed. Trans fatty acids are known to have detrimental effects on health such as increased levels of low density lipoprotein cholesterol (LDL-C) and increased risk of atherosclerosis (Han et al., 2002). Additionally, in the fastfood industry, a batch of frying oil is repeatedly reused for up to 1 to 4 days, depending on the hours of operation (Phiri, Mumba \& Mangwera, 2006). Monitoring of the frying performance of frying oils is therefore very important for human health and nutrition.

Soybean oil contains a high percentage of PUFAs (e.g. LA and ALA), which are of significant dietary importance. Gerde et al. (2007) observed that both LA and ALA in SO decreased after using the same oil to deep fry potato fries for 23 days. To minimise the undesired degradation compounds and/ or to maintain the quality of the fried products, oils that consist mostly of SFAs and MUFAs are normally chosen for deep frying (Choe \& Min, 2007). Canola is one of the five major oilseeds produced in the world (Gunstone, 2002) and the fatty acid composition of $\mathrm{CO}$ makes it one of the most ideal vegetable oil in terms of imparting health benefits. $\mathrm{CO}$ has a high percentage of monounsaturated fatty acids (MUFAs), which has higher thermal oxidative stability than PUFAs. However, a study has shown that repeated usage of $\mathrm{CO}$ for deep-frying purposes reduced the amount of MUFAs (Santos et al., 2018).

To improve frying stability, new techniques such as oil blending, hermetic frying, and addition of antioxidants into oil (Aladedunye \& Przybylski, 2009b;
Aydeniz \& Yilmaz, 2016; Choe \& Min, 2007) have been developed in recent years. Studies have shown that blending PUFA oils with SFA or MUFA oils improved the oil stability of PUFA oils (Bhatnagar et al., 2009; HashempourBaltork et al., 2016; Serjouie et al., 2010). Hence, blending two oils together might produce an end-product that has high nutritional value and oxidative stability. Farhoosh, Kenari \& Poorazrang (2009) showed that blending $\mathrm{CO}$ with other oils such as palm oil, corn oil and olive oil improved the frying stability of CO.

During deep frying of potato fries, a mass transfer between the potato fries and the oil would occur (Dobarganes, Márquez-Ruiz \& Velasco, 2000). There would be oil absorption and water loss from the potato fries. Since the potato fries would be consumed, it would be of interest to look at its fatty acid composition. Therefore, the aim of the present study was to examine: (1) the fatty acid composition changes of $\mathrm{SO}, \mathrm{CO}$, and a 1:1 blend of soybean oil and canola oil (SCO) throughout an intermittent frying process of 80 batches in five days, (2) the fatty acid composition changes in the potato fries fried in these three different types of oil.

\section{MATERIALS AND METHODS}

\section{Preparation of standard solutions for gas chromatography-mass spectrometer (GC-MS) analysis}

The internal standard solution was prepared by dissolving $200 \mathrm{mg}$ of methyl undecanoate (C11:0 FAME, Sigma Aldrich, Singapore) in $1 \mathrm{~mL}$ of n-hexane. The Fatty Acid Methyl Esters (FAMEs) calibration standard solution was prepared by dissolving $40 \mathrm{mg}$ of calibration standard (GLC 603B, NuChek-Prep, USA) in $1 \mathrm{~mL}$ of $\mathrm{n}$-hexane.

\section{Frying protocol}

The frying protocol involved intermittent 
Table 1. Fatty acid composition (\%) of Soybean Oil (SO), Canola Oil (CO) and Soybean-Canola Oil (SCO)

\begin{tabular}{|c|c|c|c|c|c|c|c|}
\hline \multirow{2}{*}{ Type of oil } & \multirow{2}{*}{ Fatty acids } & \multicolumn{6}{|c|}{ Time (h) } \\
\hline & & 0.0 & 0.8 & 1.6 & 2.4 & 3.2 & 4.0 \\
\hline \multirow[t]{14}{*}{$\mathrm{SO}$} & $\mathrm{C} 8: 0$ & 0.00 & 0.04 & 0.10 & 0.14 & 0.19 & 0.21 \\
\hline & $\mathrm{C} 14: 0$ & 0.10 & 0.10 & 0.12 & 0.11 & 0.11 & 0.12 \\
\hline & C16:0 & 13.2 & 13.4 & 13.7 & 16.2 & 15.1 & 14.4 \\
\hline & C16:1 & 0.09 & 0.10 & 0.10 & 0.12 & 0.10 & 0.11 \\
\hline & C17:0 & 0.12 & 0.12 & 0.12 & 0.13 & 0.12 & 0.13 \\
\hline & C18:0 & 5.65 & 5.81 & 5.97 & 6.00 & 6.05 & 6.29 \\
\hline & C18:1t & 0.00 & 0.00 & 0.00 & 0.07 & 0.08 & 0.10 \\
\hline & C18:1 & 25.2 & 25.5 & 25.9 & 25.7 & 26.2 & 26.4 \\
\hline & C18:2 & 46.3 & 45.8 & 45.1 & 43.1 & 43.7 & 43.9 \\
\hline & C20:0 & 0.47 & 0.49 & 0.51 & 0.52 & 0.52 & 0.57 \\
\hline & C18:3 & 7.87 & 7.63 & 7.28 & 6.86 & 6.55 & 6.55 \\
\hline & $\mathrm{C} 20: 1$ & 0.24 & 0.28 & 0.29 & 0.29 & 0.28 & 0.31 \\
\hline & $\mathrm{C} 22: 0$ & 0.48 & 0.49 & 0.51 & 0.52 & 0.52 & 0.60 \\
\hline & $\mathrm{C} 24: 0$ & 0.14 & 0.13 & 0.16 & 0.17 & 0.25 & 0.18 \\
\hline \multirow[t]{13}{*}{$\mathrm{CO}$} & $\mathrm{C} 8: 0$ & 0.00 & 0.02 & 0.06 & 0.08 & 0.11 & 0.14 \\
\hline & C16:0 & 6.15 & 6.21 & 6.32 & 6.27 & 6.38 & 6.41 \\
\hline & C16:1 & 0.26 & 0.26 & 0.27 & 0.26 & 0.26 & 0.27 \\
\hline & C18:0 & 2.52 & 2.55 & 2.61 & 2.59 & 2.66 & 2.65 \\
\hline & C18:1t & 0.00 & 0.00 & 0.09 & 0.12 & 0.17 & 0.18 \\
\hline & C18:1 & 58.2 & 58.8 & 59.4 & 60.4 & 60.6 & 61.2 \\
\hline & C18:2 & 21.3 & 21.0 & 20.5 & 20.1 & 19.8 & 19.5 \\
\hline & $\mathrm{C} 20: 0$ & 0.86 & 0.87 & 0.90 & 0.89 & 0.92 & 0.91 \\
\hline & C18:3 & 8.13 & 7.67 & 7.19 & 6.68 & 6.40 & 6.05 \\
\hline & C20: 1 & 1.75 & 1.77 & 1.80 & 1.76 & 1.80 & 1.77 \\
\hline & $\mathrm{C} 22: 0$ & 0.34 & 0.35 & 0.37 & 0.37 & 0.39 & 0.39 \\
\hline & $\mathrm{C} 22: 1$ & 0.16 & 0.16 & 0.16 & 0.16 & 0.17 & 0.17 \\
\hline & $\mathrm{C} 24: 0$ & 0.11 & 0.12 & 0.14 & 0.14 & 0.15 & 0.15 \\
\hline \multirow[t]{12}{*}{$\mathrm{SCO}$} & $\mathrm{C} 8: 0$ & 0.00 & 0.05 & 0.08 & 0.11 & 0.15 & 0.17 \\
\hline & C16:0 & 9.81 & 10.0 & 10.2 & 10.7 & 10.9 & 10.6 \\
\hline & C16:1 & 0.16 & 0.18 & 0.18 & 0.18 & 0.19 & 0.17 \\
\hline & C18:0 & 4.12 & 4.26 & 4.37 & 4.30 & 4.48 & 4.56 \\
\hline & C18:1t & 0.00 & 0.00 & 0.09 & 0.11 & 0.12 & 0.15 \\
\hline & C18:1 & 40.7 & 41.7 & 42.2 & 42.6 & 42.7 & 43.1 \\
\hline & C18:2 & 35.0 & 34.2 & 33.6 & 33.2 & 32.7 & 32.6 \\
\hline & C20:0 & 0.63 & 0.68 & 0.71 & 0.69 & 0.75 & 0.76 \\
\hline & C18:3 & 8.06 & 7.30 & 6.90 & 6.44 & 6.30 & 6.14 \\
\hline & C20: 1 & 0.85 & 0.90 & 0.91 & 0.90 & 0.94 & 0.98 \\
\hline & $\mathrm{C} 22: 0$ & 0.39 & 0.41 & 0.42 & 0.42 & 0.44 & 0.47 \\
\hline & $\mathrm{C} 24: 0$ & 0.13 & 0.13 & 0.13 & 0.14 & 0.15 & 0.16 \\
\hline
\end{tabular}


frying of 80 batches of fries over five days. Fresh U.S. Russet potatoes (FairPrice NTUC, Singapore) were peeled and cut into equal lengths of 60 to 70 $\mathrm{mm}$, with an equal width and height of $12 \mathrm{~mm} .5 \mathrm{~L}$ of each type of oil, namely $\mathrm{SO}, \mathrm{CO}$ and $\mathrm{SCO}$, was used for frying. For SCO, $2.5 \mathrm{~L}$ of $\mathrm{SO}$ and $2.5 \mathrm{~L}$ of $\mathrm{CO}$ were mixed together before frying. Potato fries were fried in $100 \mathrm{~g}$ batches at $180^{\circ} \mathrm{C}$ for $3 \mathrm{~min}$. Batches of fries were fried at 10-min intervals and 16 batches were processed per day for five consecutive days. Oil samples were taken on the first day before frying and after every four batches of frying. At the end of each day, the fryer was turned off and the oil was cooled to room temperature.

\section{Lipid extraction}

Lipid in the potato fries was extracted with petroleum spirit $\left(40^{\circ} \mathrm{C}-60^{\circ} \mathrm{C}\right)$ using the Soxtec 2055 system (FOSS, Denmark). The extraction method used in this study was adapted and modified from Matsler \& Siebenmorgen (2005). $5 \mathrm{~g}$ of each sample was weighed into the cellulose thimbles and a thin layer of defatted cotton was placed on top of the sample. The thimbles containing the samples were then placed in the oven at $103 \pm 2{ }^{\circ} \mathrm{C}$ for $2 \mathrm{~h}$. Next, the thimbles were placed into the Soxtec system and the extraction cups were placed beneath the thimbles. The Soxtec extraction programme consisted of four steps, which were boiling, rinsing, recovery and pre-drying. Firstly, the samples were immersed in boiling petroleum spirit for $30 \mathrm{~min}$ and then rinsed for 40 min. Next, there was a recovery stage of $10 \mathrm{~min}$, followed by pre-drying for $5 \mathrm{~min}$. The hotplate temperature was held at $135^{\circ} \mathrm{C}$ throughout the four stages. After extraction, the cups were dried at $103 \pm$ $2^{\circ} \mathrm{C}$ for $30 \mathrm{~min}$ and then placed in the dessicator. The fat obtained from each sample was stored at $-20^{\circ} \mathrm{C}$ until further analysis.

\section{Determination of fatty acid composition}

Determination of fatty acid composition was based on an application note by Agilent (Juskelis et al., 2014). Oil samples were diluted in a 1:1 ratio with hexane. $10 \mu \mathrm{L}$ of the $1: 1$ mixture and $3.3 \mu \mathrm{L}$ of internal standard $(200 \mathrm{mg} / \mathrm{mL})$ were added into a gas chromatography (GC) vial. Next, the GC vial was placed on the sample tray of the GC autosampler. Derivatisation of fatty acids was performed on the Agilent Sample Prep WorkBench and the steps for the derivatisation were as follows: Firstly, $120 \mu \mathrm{L}$ of $2 \mathrm{~N}$ sodium hydroxide $(\mathrm{NaOH})$ in methanol was added to the vial and mixed for $20 \mathrm{~s}$ at $1500 \mathrm{rpm}$. Then, the vial was transferred to the heater at $70^{\circ} \mathrm{C}$ for $5 \mathrm{~min}$. Samples were allowed to cool for $5 \mathrm{~min}$ before $240 \mu \mathrm{L}$ of $12.5 \%$ boron trifluoride $\left(\mathrm{BF}_{3}\right)$ in methanol was added. The vial was mixed for $20 \mathrm{~s}$ at $1500 \mathrm{rpm}$ and transferred to the heater at $70^{\circ} \mathrm{C}$ for 5 min. Samples were allowed to cool for $5 \mathrm{~min}$ before $300 \mu \mathrm{L}$ of water and 300 $\mu \mathrm{L}$ of hexane were added. The vial was mixed for $20 \mathrm{~s}$ at $1500 \mathrm{rpm}$ and the top layer $(1 \mu \mathrm{L})$ was injected into the GC-MS.

\section{GC-MS analysis}

The analysis was performed using a 7890B GC system (Agilent Technologies, USA) coupled with the MS detector with MSD ChemStation software. The GC-MS parameters were adapted from Agilent (Juskelis et al., 2014). The separation of the different fatty acid methyl esters was performed on a HP-88 column $(60 \mathrm{~m} \times$ $0.25 \mathrm{~mm}, 0.20 \mu \mathrm{m}$, Agilent Technologies, USA). A split injector at $250^{\circ} \mathrm{C}$ and a spilt ratio of 50:1 was used. The oven temperature programme was isothermal at $140^{\circ} \mathrm{C}$ for $5 \mathrm{~min}$. The temperature was increased to $240^{\circ} \mathrm{C}$ at a rate of $4^{\circ} \mathrm{C} / \mathrm{min}$ and maintained for 0 min. Helium was used as the carrier gas under a constant flow mode at $1 \mathrm{~mL} / \mathrm{min}$. The MS detector was at $280^{\circ} \mathrm{C}$ and a scan acquisition 
mode of 40 to 500 AMU was used. The FAMEs were identified by comparing their retention times and mass spectrum with the calibration standards. In this study, peaks that cannot be identified were not considered in the calculation of the percentage of fatty acids.

\section{RESULTS}

The fatty acid profile of SO before frying is shown in Table 1 . The major fatty acids in SO included C16:0 (palmitic acid or PA, 13.21\%), C18:0 (stearic acid or SA, 5.65\%), C18:1 (oleic acid or OA, 25.23\%), C18:2 (LA, 46.28\%) and C18:3 (ALA, 7.87\%). No trans fatty acid (C18:1t) was observed in pure SO. As seen from Table 1, changes in the fatty acid composition profile of SO were observed during continuous frying process. It was noticed that the levels of C18:2 (LA) and C18:3 (ALA) in frying oil decreased, while the levels of other fatty acids increased with frying time. At the end of 4-h frying, the levels of LA and ALA decreased by $2.39 \%$ and $1.32 \%$, respectively. Similarly, as shown in Table 1 , the most abundant fatty acid in $\mathrm{CO}$ was $\mathrm{C} 18: 1$ (OA, 58.23\%). Other major fatty acids included C16 (PA, 6.15\%), C18 (SA,

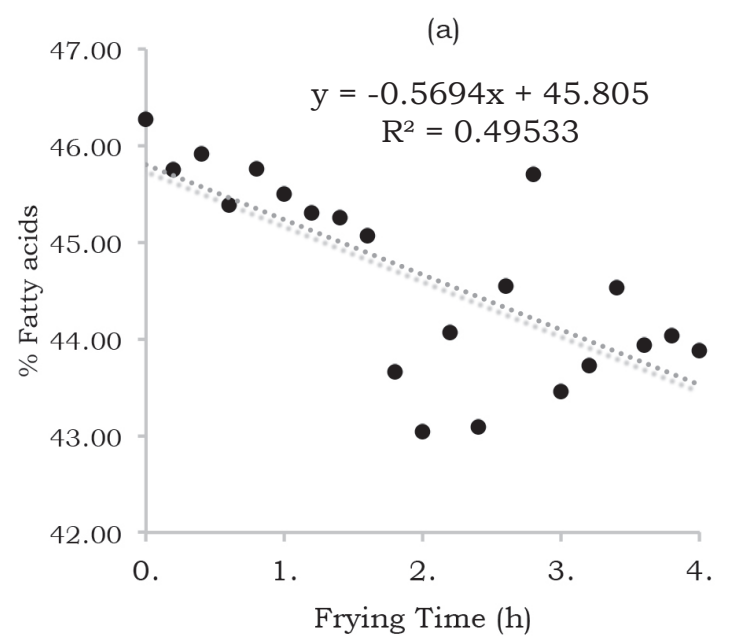

2.52\%), C18:2 (LA, 21.29\%) and C18:3 (ALA, 8.13\%). Similar to SO, deep-frying potatoes in $\mathrm{CO}$ led to the decrease of LA and ALA, but to an increase of other fatty acids. At the end of $4 \mathrm{~h}$ frying, the levels of LA and ALA decreased by $1.84 \%$ and $2.08 \%$, respectively. When SO and $\mathrm{CO}$ was blended $(\mathrm{SCO}, \mathrm{v} / \mathrm{v}=1: 1)$, Table 1 shows that the main fatty acids in SCO were OA (40.69\%) and LA (34.95\%). At the end of $4 \mathrm{~h}$ frying, the levels of LA and ALA decreased by $2.39 \%$ and $1.92 \%$, respectively. On the other hand, the contents of trans fatty acid (C18:1t) increased by $0.10 \%, 0.18 \%$, and $0.15 \%$ during frying time in $\mathrm{SO}, \mathrm{CO}$, and $\mathrm{SCO}$, respectively.

To evaluate the kinetic rate for LA and ALA degradation, the levels of LA and ALA at different frying time in SO are shown in Figures $1 \mathrm{a}$ and $1 \mathrm{~b}$, respectively. In this system, the decrease of LA can be described by:

$$
r=-\frac{d[L A]}{d t}=k[L A]^{n}
$$

where $k$ is the rate constant or rate coefficient and $n$ is the reaction order.

Figure 1a shows that $[L A]=45.805-$ $0.5694 \times t$.

(b)

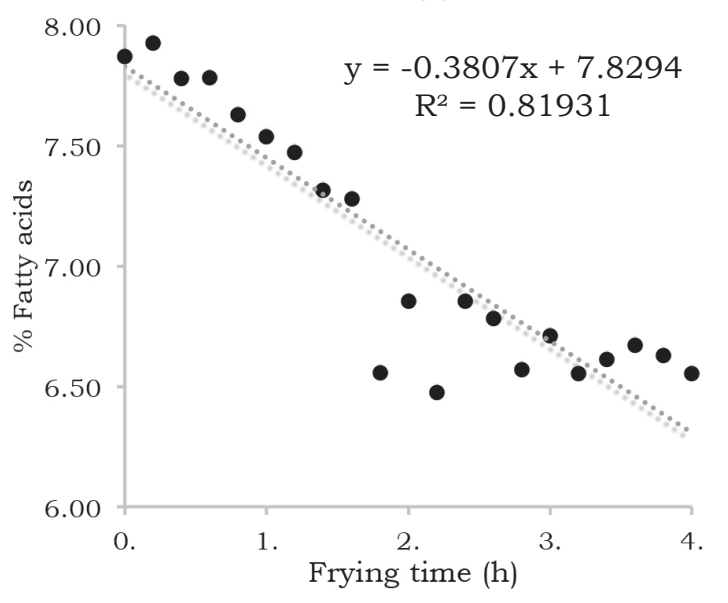

Figure 1(a) Changes in LA with frying time for SO;(b) Changes in ALA with frying time for SO 
(a)

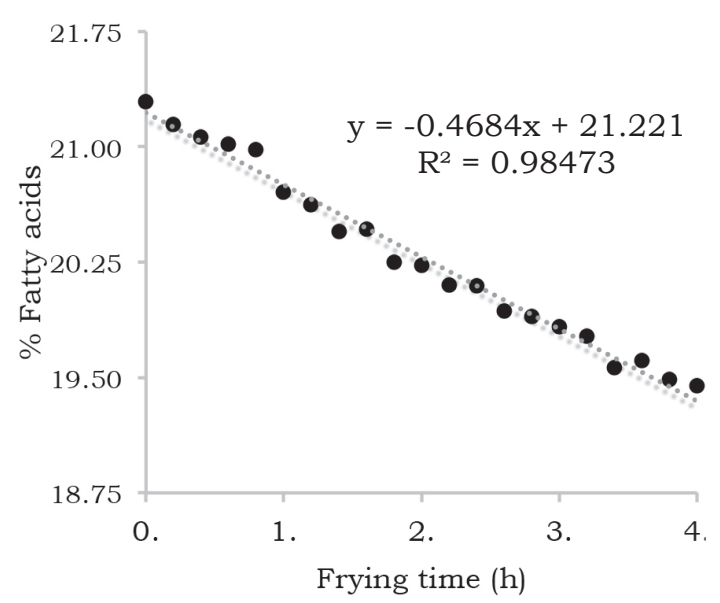

(b)

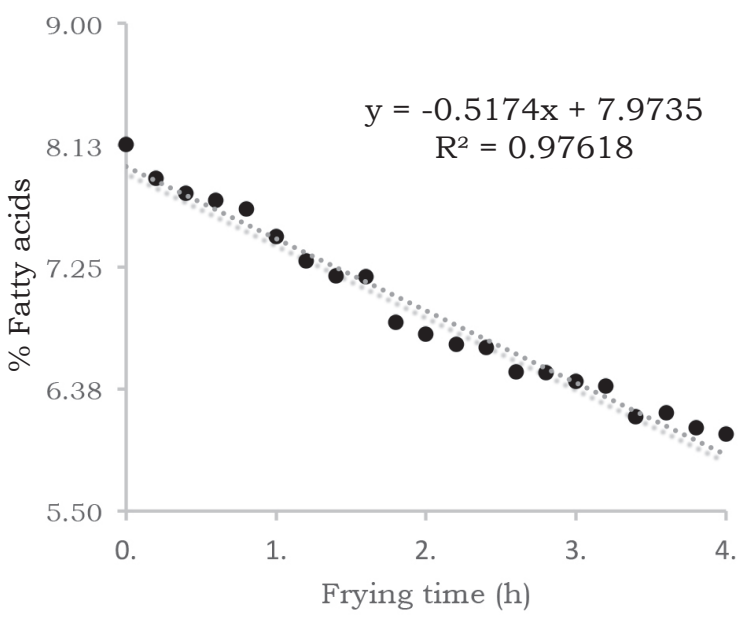

Figure 2(a) Changes in LA with frying time for CO; (b) Changes in ALA with frying time for CO

(a)

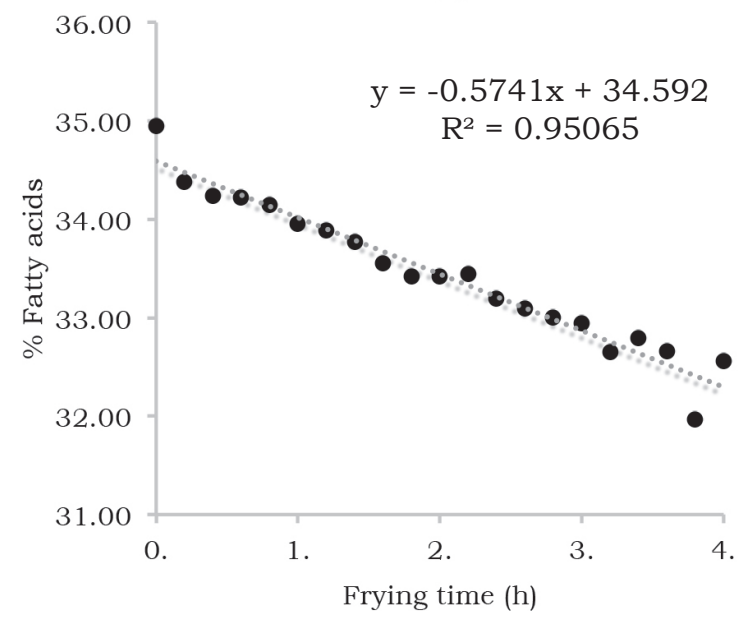

(b)

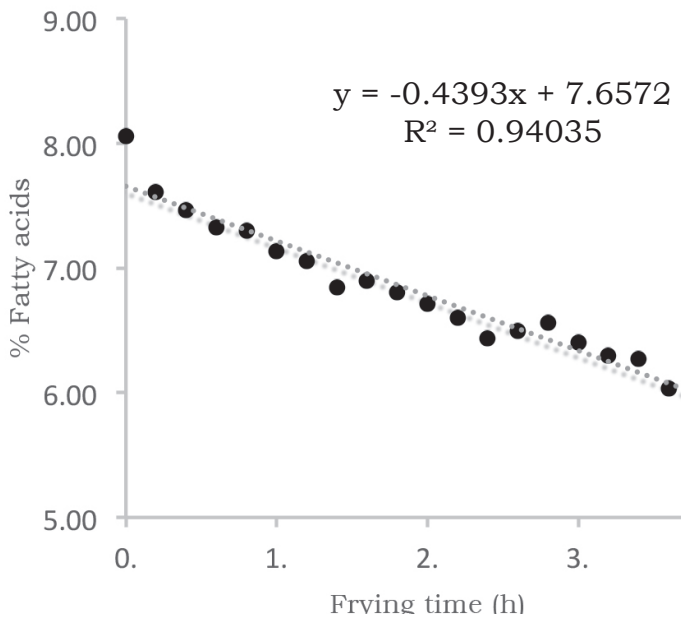

Figure 3(a) Changes in LA with frying time for SCO; (b) Changes in ALA with frying time for SCO

Therefore, the degradation of LA during deep-frying is a pseudo-zeroorder reaction with a reaction constant of $0.569 \mathrm{~h}^{-1}$. Similar reaction orders were observed for LA in the other two oils (Figures $2 \mathrm{a}$ and $3 \mathrm{a}$ ), as well as ALA in all three oils (Figures $1 \mathrm{~b}, 2 \mathrm{~b}$, and $3 \mathrm{~b}$ ). Based on the reaction orders, the halflife of LA and ALA, which is the time needed for the percentage of LA and ALA in the oil to decrease to half of its original value, was calculated. For SO, it would take $40.2 \mathrm{~h}$ for LA and $10.3 \mathrm{~h}$ for ALA, respectively to decrease to half of their original values. While for $\mathrm{CO}$, it would take $22.7 \mathrm{~h}$ and $7.7 \mathrm{~h}$ for LA and ALA, respectively to decrease to half of their original values. In $\mathrm{SCO}$, the halflife of LA and ALA were $30.1 \mathrm{~h}$ and $8.7 \mathrm{~h}$, respectively.

Fatty acid compositions and trans fatty acid contents of potato fries fried in 
Table 2. Fatty acid composition (\%) of potato fries fried in Soybean Oil (SO), Canola Oil (CO) and Soybean-Canola Oil (SCO)

\begin{tabular}{|c|c|c|c|c|c|c|}
\hline \multirow{2}{*}{ Type of oil } & \multirow{2}{*}{ Fatty acids } & \multicolumn{5}{|c|}{ Time (h) } \\
\hline & & 0.8 & 1.6 & 2.4 & 3.2 & 4.0 \\
\hline \multirow[t]{14}{*}{$\mathrm{SO}$} & $\mathrm{C} 8: 0$ & 0.06 & 0.09 & 0.17 & 0.21 & 0.25 \\
\hline & C14:0 & 0.10 & 0.11 & 0.12 & 0.13 & 0.13 \\
\hline & C16:0 & 13.7 & 14.0 & 15.3 & 15.7 & 15.8 \\
\hline & C16:1 & 0.10 & 0.09 & 0.12 & 0.12 & 0.12 \\
\hline & $\mathrm{C} 17: 0$ & 0.11 & 0.12 & 0.13 & 0.14 & 0.14 \\
\hline & C18:0 & 5.57 & 6.00 & 6.86 & 6.72 & 6.89 \\
\hline & C18:1t & 0.00 & 0.00 & 0.07 & 0.09 & 0.10 \\
\hline & C18:1 & 25.8 & 26.0 & 28.1 & 27.8 & 28.1 \\
\hline & C18:2 & 46.7 & 45.0 & 41.9 & 41.5 & 41.2 \\
\hline & C20:0 & 0.45 & 0.51 & 0.58 & 0.61 & 0.61 \\
\hline & C18:3 & 6.62 & 7.15 & 5.48 & 5.71 & 5.37 \\
\hline & C20: 1 & 0.23 & 0.27 & 0.31 & 0.31 & 0.33 \\
\hline & $\mathrm{C} 22: 0$ & 0.43 & 0.55 & 0.63 & 0.57 & 0.66 \\
\hline & $\mathrm{C} 24: 0$ & 0.13 & 0.14 & 0.18 & 0.20 & 0.19 \\
\hline \multirow[t]{13}{*}{$\mathrm{CO}$} & $\mathrm{C} 8: 0$ & 0.04 & 0.06 & 0.09 & 0.11 & 0.15 \\
\hline & C16:0 & 7.14 & 6.20 & 6.72 & 6.68 & 6.73 \\
\hline & C16:1 & 0.27 & 0.25 & 0.29 & 0.28 & 0.26 \\
\hline & C18:0 & 2.74 & 2.58 & 2.84 & 2.81 & 2.77 \\
\hline & C18:1t & 0.00 & 0.08 & 0.12 & 0.15 & 0.16 \\
\hline & C18:1 & 59.4 & 59.4 & 60.4 & 60.9 & 62.0 \\
\hline & C18:2 & 20.2 & 20.8 & 19.6 & 19.5 & 19.0 \\
\hline & C20:0 & 0.87 & 0.87 & 0.94 & 0.92 & 0.89 \\
\hline & C18:3 & 6.71 & 7.14 & 6.12 & 5.92 & 5.37 \\
\hline & C20: 1 & 1.75 & 1.73 & 1.84 & 1.82 & 1.77 \\
\hline & $\mathrm{C} 22: 0$ & 0.37 & 0.37 & 0.43 & 0.42 & 0.39 \\
\hline & C22: 1 & 0.18 & 0.18 & 0.20 & 0.18 & 0.17 \\
\hline & $\mathrm{C} 24: 0$ & 0.14 & 0.14 & 0.15 & 0.15 & 0.15 \\
\hline \multirow[t]{13}{*}{$\mathrm{SCO}$} & C8:0 & 0.07 & 0.09 & 0.12 & 0.16 & 0.20 \\
\hline & C16:0 & 10.8 & 10.7 & 10.7 & 10.8 & 11.1 \\
\hline & C16:1 & 0.20 & 0.19 & 0.19 & 0.19 & 0.20 \\
\hline & C17:0 & 0.10 & 0.10 & 0.10 & 0.10 & 0.10 \\
\hline & C18:0 & 4.55 & 4.72 & 4.73 & 4.77 & 4.97 \\
\hline & C18:1t & 0.08 & 0.09 & 0.11 & 0.14 & 0.17 \\
\hline & C18:1 & 43.2 & 42.8 & 42.9 & 43.0 & 43.3 \\
\hline & C18:2 & 32.5 & 32.4 & 32.4 & 32.2 & 31.5 \\
\hline & C20:0 & 0.77 & 0.77 & 0.77 & 0.78 & 0.82 \\
\hline & C18:3 & 5.96 & 6.35 & 6.20 & 6.14 & 5.80 \\
\hline & C20: 1 & 0.95 & 0.97 & 0.98 & 0.99 & 1.03 \\
\hline & $\mathrm{C} 22: 0$ & 0.47 & 0.47 & 0.44 & 0.49 & 0.54 \\
\hline & $\mathrm{C} 24: 0$ & 0.18 & 0.18 & 0.17 & 0.18 & 0.19 \\
\hline
\end{tabular}


SO are shown in Table 2. By comparison of Table 1 and 2, the fatty acid profiles of $\mathrm{SO}$ and potato fries fried in SO were similar, but the decrease of LA in potato fries after 4-h frying was greater than that in SO $(5.54 \%$ vs $2.39 \%)$. The fatty acid profiles of potato fries fried in $\mathrm{CO}$ and SCO shown in Table 2 were also similar to those in oils (Table 1). Unlike the results obtained in $\mathrm{SO}$, the fries fried in CO had a smaller ALA decrease after 4-h frying than ALA decrease in the pure oil (1.34\% vs $2.08 \%)$, as shown in Table 1 and 2 . Table 1 and 2 also showed that the decrease of ALA was even smaller in fries fried in SCO than that in SCO $(0.16 \%$ vs $1.92 \%)$.

\section{DISCUSSION}

In this paper, we examined the frying performance of potato fries in two traditional oils, i.e. SO (the most abundant fatty acid is LA) and $\mathrm{CO}$ (the most abundant fatty acid is OA). The fatty acid profiles of $\mathrm{SO}$ and $\mathrm{CO}$ before frying were in agreement with other studies (Abdulkarim et al., 2007; Dubois et al., 2007). Throughout $4 \mathrm{~h}$ of frying, LA in SO decreased by $2.39 \%$ whereas LA in $\mathrm{CO}$ decreased by $1.84 \%$. In contrast, ALA in SO decreased by $1.32 \%$, whereas ALA in CO decreased by $2.08 \%$. The small degradation of LA and ALA in the present study was probably due to the lower frying temperature (i.e. $180^{\circ} \mathrm{C}$ ). The percentage decrease of LA and ALA in both SO and $\mathrm{CO}$ were similar. Based on the percentage decrease of LA and ALA, our results suggest that oils containing PUFAs were as good as those containing MUFAs in deep-frying process.

Previous studies have shown that blending PUFA oils with SFA or MUFA oils improved the oil stability of PUFA oils (Bhatnagar et al., 2009; Hashempour-Baltork et al., 2016; Serjouie et al., 2010). In this study, we examined the frying performance of
SCO because there is little literature that investigates the fatty acid profile changes in SCO (Hashempour-Baltork et al., 2016). Based on Table 1, it was observed that the levels of LA and ALA in SCO decreased by $2.39 \%$ and $1.92 \%$, respectively. However, the levels of LA and ALA in SO decreased by $2.39 \%$ and $1.32 \%$, respectively. The blending of CO and SO did not significantly improve the degradation levels of LA and ALA in SCO during the deep-frying process. During the deep-frying process, there was also formation of C18:1t in SCO. The levels of C18:1t in SCO increased by $0.15 \%$, which was lower compared to the $0.18 \%$ increase of $\mathrm{C} 18: 1 \mathrm{t}$ in CO. However, the increase of $\mathrm{C} 18: 1 \mathrm{t}$ in SCO was still larger than the increase of $\mathrm{C} 18: 1 \mathrm{t}$ in $\mathrm{SO}$ $(0.10 \%)$. Hence, our results suggest that blending soybean oil and canola oil does not improve the frying stability of the resulting oil in terms of fatty acid profile.

Interestingly, it was observed that the contents of octanoic acid (C8:0) increased with frying time. This result was in agreement with another study conducted by Aladedunye \& Przybylski (2009a), where the levels of octanoic acid increased with frying time. MarquezRuiz \& Dobarganes (1996) mentioned that the quantification of octanoic acid and heptanoic acid has been used as an assay for oxidative deterioration in fats and oils that did not contain naturally occurring octanoic acid and heptanoic acid. This is because the oxidation of unsaturated fatty acyl radicals produces a radical attached to the glyceridic backbone. After the addition of $\mathrm{H}$., a bound octanoic acid or heptanoic acid attached to the glyceridic backbone will be formed. After the transesterification process, octanoic acid and heptanoic acid will be released. The oils used in this study contained OA, LA, and ALA, which were able to form octanoic acid from the process described above. As the 
frying time increases, the oils undergo oxidation and hydrolysis, which lead to the elevation of octanoic acid. Heptanoic acid formation was not observed in this study due to the absence of heptanoic acid methyl ester in the calibration standards.

We also observed that the levels of OA, PA, and SA increased with frying time. Our results are consistent with a previous study showing that SFAs increased in both rapeseed oil and SO as the number of frying cycles increased (Bhardwaj et al., 2016). Similarly, Gerde et al. (2007) observed that OA, PA, and $\mathrm{SA}$ in SO increased as the number of frying cycles increased. The increase in SFAs (PA and SA) and OA might be due to the breakdown of LA and ALA, which could transform into fatty acids with the same number of carbons or with lesser number of carbons.

During the deep-frying process, there was the formation of C18:1 trans fatty acid as the number of frying cycles increased. Fatty acids in oils heated at high temperatures are known to undergo chemical conversion from cis to trans isomer. Trans fatty acids in frying oils can be produced from free radical reaction, heat-induced isomerisation or concerted reaction (Zhang et al., 2012). Tsuzuki et al. (2008) concluded that heating of highly purified triolein, trilinolein and trilinolenin induced cis to trans isomerisation. As the heating period increases, the amount of trans fatty acid increases. Bhardwaj et al. (2016) also reported that the amount of trans fatty acids in rapeseed oil and soybean oil increased as the number of frying cycles increased. Tsuzuki et al. (2008) mentioned that lipid oxidation of unsaturated fatty acids occurs together with the geometric isomerisation of unsaturated fatty acids. This is because trans-isomerism and polar compounds were not detected when triolein with added $\alpha$-tocopherol was heated under a nitrogen stream. Hence, the formation of C18:1 trans fatty acid might have contributed to the decrease in linoleic and linolenic acids.

In this study, the fatty acid composition of the potato fries was similar to the fatty acid composition of the oils. This is due to the mass transfer that occurred during deep-frying of the potato fries. Mousa (2018) observed that oil absorption of potato fries occurred during deep-frying. Hence, the potato fries would have a similar fatty acid composition as the oil it was fried in.

\section{CONCLUSION}

Repeated usage of frying oils resulted in a decrease in the amount of PUFAs, i.e. LA and ALA and an increase in the amount of MUFAs and SFAs in the oils. After $4 \mathrm{~h}$ of frying, the levels of LA and ALA in SO decreased by $2.39 \%$ and $1.32 \%$ respectively, while for $\mathrm{CO}$, the levels of LA and ALA decreased by $1.84 \%$ and $2.08 \%$ respectively. For SCO, LA and ALA decreased by $2.39 \%$ and $1.92 \%$ respectively after $4 \mathrm{~h}$ of frying. As for trans fatty acids, the levels of C18:1t increased by $0.10 \%$ for SO, while the levels of $\mathrm{C} 18: 1 \mathrm{~T}$ increased by $0.18 \%$ for CO. For SCO, the levels of C18:1T increased by $0.15 \%$. In SCO, the halflife of LA and ALA were found to be $30.1 \mathrm{~h}$ and $8.7 \mathrm{~h}$, respectively. Hence, blending soybean oil and canola oil did not significantly improve the fatty acid profile of the resulting oil. As for the potato fries, the fatty acid composition was consistent with the fatty acid composition of the vegetable oils. Due to the formation of trans fatty acids and the decrease in PUFAs, it is advised not to use the same frying oil repeatedly and not to consume food products cooked in reused oil. 


\section{Acknowledgement}

The authors greatly acknowledge the financial support from A*STAR BMRC (Biomedical Research Council) and this research was funded by IAF-PP (HBMS Domain): H17/01/a0/A11 Food Structure Engineering for Nutrition and Health-CNRC Core Funds.

\section{Authors' contributions}

MTYY, conducted the study, data analysis and interpretation, prepared the draft of the manuscript; XB, conducted data analysis and interpretation, reviewed the manuscript; $\mathrm{CJH}$, principal investigator, conceptualised and designed the study, reviewed the manuscript.

\section{Conflicts of interest}

We declare no conflict of interests.

\section{References}

Abdulkarim SM, Long K, Lai OM, Muhammad SKS \& Ghazali HM (2007). Frying quality and stability of high-oleic Moringa oleifera seed oil in comparison with other vegetable oils. Food Chem 105(4):1382-1389.

Aladedunye FA \& Przybylski R (2009a). Degradation and nutritional quality changes of oil during frying. J Am Oil Chem Soc 86(2):149-156.

Aladedunye FA \& Przybylski R (2009b). Protecting oil during frying: A comparative study. Eur $J$ Lipid Sci Technol 111(9):893-901.

Alvis A, Vélez C, Rada-Mendoza M, Villamiel M \& Villada HS (2009). Heat transfer coefficient during deep-fat frying. Food Control 20(4):321325.

Aydeniz B \& Yilmaz E (2016). Performance of different natural antioxidant compounds in frying oil. Food Technol Biotechnol 54(1):21-30.

Bhatnagar AS, Prasanth Kumar PK, Hemavathy J \& Gopala Krishna AG (2009). Fatty acid composition, oxidative stability, and radical scavenging activity of vegetable oil blends with coconut oil. J Am Oil Chem Soc 86(10):991-999.

Bhardwaj S, Passi SJ, Misra A, Pant KK, Anwar K, Pandey RM \& Kardam V (2016). Effect of heating/reheating of fats/oils, as used by Asian Indians, on trans fatty acid formation. Food Chem 212:663-670.

Calder PC (2006). n-3 Polyunsaturated fatty acids, inflammation, and inflammatory diseases. Am $J$ Clin Nutr 83(6):1505S-1519S.

Choe E \& Min DB (2007). Chemistry of deep-fat frying oils. J Food Sci 72(5):77-86.
Dobarganes C, Márquez-Ruiz G \& Velasco J (2000). Interactions between fat and food during deepfrying. Eur J Lipid Sci Technol 102(8-9):521528.

Dubois V, Breton S, Linder M, Fanni J \& Parmentier M (2007). Fatty acid profiles of 80 vegetable oils with regard to their nutritional potential. Eur $J$ Lipid Sci Technol 109(7):710-732.

Farhoosh R, Kenari RE \& Poorazrang H (2009). Frying stability of canola oil blended with palm olein, olive, and corn oils. J Am Oil Chem Soc 86(1):71-76.

Gerde J, Hardy C, Fehr W \& White PJ (2007). Frying performance of no-trans, low-linolenic acid soybean oils. J Am Oil Chem Soc 84(6):557563.

Gunstone FD (2002). Vegetable oils in food technology: composition, properties and Uses. Blackwell Publishing Ltd., New Jersey.

Han SN, Leka LS, Lichtenstein AH, Ausman LM, Schaefer EJ \& Meydani SN (2002). Effect of hydrogenated and saturated, relative to polyunsaturated, fat on immune and inflammatory responses of adults with moderate hypercholesterolemia. $J$ Lipid Res 43:445-452.

Hashempour-Baltork F, Torbati M, AzadmardDamirchi S \& Savage GP (2016). Vegetable oil blending: A review of physicochemical, nutritional and health effects. Trends Food Sci Technol 57:52-58.

Juskelis R, Cappozzo J, Jablonski J, Wylie PL \& Mrozinski P (2014). Automated Sample Preparation for FAME Analysis in Edible Oils Using an Agilent 7696A Sample Prep WorkBench. From https://www.agilent.com/ cs/library/applications /5991-5172EN-D2.pdf [Retrieved January 14 2019].

Marquez-Ruiz G \& Dobarganes C (1996). Short-chain fatty acid formation during thermoxidation and frying. $J$ Sci Food Agric 70(1): 120-126.

Matsler AL \& Siebenmorgen TJ (2005). Evaluation of operating conditions for surface lipid extraction from rice using a Soxtec System. Cereal Chem 82(3):282-286.

Mousa RMA (2018). Simultaneous inhibition of acrylamide and oil uptake in deep fat fried potato strips using gum Arabic-based coating incorporated with antioxidants extracted from spices. Food Hydrocoll 83:265-274. 
Phiri G, Mumba P \& Mangwera A (2006). The quality of cooking oil used in informal food processing in Malawi: a preliminary study. Int J Consum Stud 30(6):527-532.

Rajaram S (2014). Health benefits of plantderived $\alpha$-linolenic acid. Am $J$ Clin Nutr $100(1): 443$ S-448S.

Santos CSP, Molina-Garciaa L, Cunhaa SC \& Casal S (2018). Fried potatoes: Impact of prolonged frying in monounsaturated oils. Food Chem 243:192-201.

Serjouie A, Tan CP, Mirhosseini H \& Che Man BY (2010). Effect of vegetable-based oil blends on physicochemical properties of oils during deepfat frying. Am J Food Technol 5(5):310-323.
Tsuzuki W, Nagata R, Yunoki R, Nakajima M \& Nagata T (2008). cis/trans-Isomerisation of triolein, trilinolein and trilinolenin induced by heat treatment. Food Chem 108(1):75-80.

Zhang Q, Saleh ASM, Chen J \& Shen Q (2012). Chemical alterations taken place during deepfat frying based on certain reaction products: A review. Chem Phys Lipids 165(6):662-681. 